\title{
Analysis of Resistive Superconducting Transition of a $\left(\mathrm{Tl}_{0.6} \mathrm{~Pb}_{0.24} \mathrm{Bi}_{0.16}\right)\left(\mathrm{Ba}_{0.1} \mathrm{Sr}_{0.9}\right)_{2} \mathrm{Ca}_{2} \mathrm{Cu}_{3} \mathrm{O}_{y}$ Film
}

\author{
W.M. Woch*, M. Chrobak And A. KoŁodzIEjCZyK \\ Solid State Physics Department, Faculty of Physics and Applied Computer Science \\ AGH University of Science and Technology, al. Mickiewicza 30, 30-059 Kraków, Poland
}

\begin{abstract}
In this paper we analyze the resistive transition as a function of both the temperature and the dc applied magnetic field. We use the two models: first, based on the Ambegaokar and Halperin theory that describes the resistive transitions by the modified Bessel function and second, based on the Anderson and Kim theory represented by the exponential formula. The fits of the models to experimental results show that the freezing into superconducting vortex-glass phase takes place at the same temperature that does not practically depend on the applied magnetic field.
\end{abstract}

PACS numbers: 74.25.Uv, 74.25.Sv, 74.20.De

\section{Introduction}

The resistive transition from a normal to the superconducting state of high temperature superconductors (HTS) is always significantly broadened as compared to the low temperature superconductors, especially, when the applied magnetic field and/or the flowing current is present. The field-broadened resistive transition may be described by the following equation [1,2]:

$$
\Delta T=C H^{m}+\Delta T_{0},
$$

where the width of the resistive transition was usually defined by the formula: $\Delta T=T_{90 \%}-T_{10 \%}$. The value of $m$ should be $2 / 3$, but was found to depend on some properties of a superconductor. $\Delta T_{0}$ is the width of the resistive transition at zero applied magnetic field and the coefficient $C$ depends on the critical current at zero magnetic field and on the critical temperature.

The measurements of the width and the shape of the resistance transition of the HTS give insight into the flux pinning properties. From these measurements one can extract such parameters of the vortex dynamics as activation energy and frequency of flux creep [3]. Koch et al. showed [4] that in epitaxial films there is a second-order phase transition between a normal and a superconducting state at a well defined temperature $T_{\mathrm{g}}$ called the freezing temperature into the superconducting vortex-glass state.

In this paper we fitted the resistive transition as a function of both the temperature and the applied magnetic field of a $\left(\mathrm{Tl}_{0.6} \mathrm{~Pb}_{0.24} \mathrm{Bi}_{0.16}\right)\left(\mathrm{Ba}_{0.1} \mathrm{Sr}_{0.9}\right)_{2} \mathrm{Ca}_{2} \mathrm{Cu}_{3} \mathrm{O}_{y}$ superconducting film on single-crystalline lanthanum aluminate substrate, using the two models: first, based on the Ambegaokar and Halperin theory $[3,5]$ that describes the resistive transitions by the modified Bessel function

\footnotetext{
* corresponding author; e-mail: wmwoch@agh.edu.pl
}

and second, based on Anderson and Kim theory [6-8] and its extensions for the critical state represented by an exponential formula.

\section{Models}

According to Ambegaokar and Halperin $[3,5]$, in the limit of small currents the resistance of each weak link treated as a Josephson junction is reduced by the ratio

$$
R / R_{0}=\left[P_{0}\left(\gamma_{0} / 2\right)\right]^{-2},
$$

where $P_{0}$ is the modified Bessel function given by the following formula:

$$
P_{0}(x)=\sum_{s=0}^{\infty} \frac{1}{(s !)^{2}}\left(\frac{x}{2}\right)^{2 s} .
$$

Thermally activated processes depend exponentially on normalized energy barrier height $U_{0} / k_{\mathrm{B}} T$ defined by:

$$
\gamma_{0}=U_{0} / k_{\mathrm{B}} T=\left[C^{*} J_{\mathrm{c} 0}(0) / T_{\mathrm{c}} H\right] g(t),
$$

where $C^{*}$ is a constant, $J_{\mathrm{c} 0}(0)$ is the critical current density at $T=0$ and $H=0$ evaluated along the crystal direction of the field in the resistance measurement and $t=T / T_{\mathrm{c}}$. The function [1]:

$$
g(t)=\frac{\left(1-t^{2}\right)\left(1-t^{4}\right)^{1 / 2}}{t} \approx 4(1-t)^{3 / 2}
$$

includes the temperature dependences of $H_{\mathrm{c}}$ and $\lambda$ in the "two-fluid" empirical approximation. In the region from $t=1$ down to $t=1 / 2$ formula (3) can be rewritten as

$$
\gamma_{0}=A(1-t)^{3 / 2} / H,
$$

where $A=4 C^{*} J_{\mathrm{c} 0} / T_{\mathrm{c}}$.

Combining formulae (2) and (4) one can write

$$
R / R_{0}=\left\{P_{0}\left[A(1-t)^{3 / 2} / 2 H\right]\right\}^{-2} .
$$

This equation has only two parameters: $A$ and $T_{\mathrm{c}}$. The onset resistance $R_{0}$ may be taken from experiment. 
According to Anderson and Kim theory [6-8] the dissipation due to flux motion in the mixed state of an epitaxial superconducting film can be well described by the thermal activation of the form [7]:

$$
R=R_{0} \exp \left(-U / k_{\mathrm{B}} T\right) .
$$

The activation energy barrier $U$ has a functional dependence on temperature $T$, magnetic field $H$ and transport current $I$ as

$$
U=A^{*} H^{-\beta} \ln \left(I / I_{0}\right) g^{*}(T),
$$

where $A^{*}$ is a constant depending weakly on applied magnetic field, $\beta$ is a constant with value close to $1, I_{0}$ is the threshold current and $g^{*}(T)$ is the temperature function given in the following relation:

$$
g^{*}(t)=\left(1-t^{2}\right)\left(1-t^{4}\right)^{1 / 2} .
$$

Combining Eqs. (6), (7) and (8) we can recast the dissipation due to thermally activated flux creep with field, current, and temperature as follows:

$$
R=R_{0}\left(\frac{I}{I_{0}}\right)^{\frac{-A^{*} H^{-\beta}\left(1-t^{2}\right)\left(1-t^{4}\right)^{p}}{k_{\mathrm{B} T}}},
$$

where we introduced parameter $p$ instead of $1 / 2$. For initial fitting some starting values of the parameters may be estimated. We observed that the flowing current up to $5 \mathrm{~mA}$ did not broaden the resistive transition and we took it as $I_{0}$. The optimal current used in these measurements is $1 \mathrm{~mA}$. Therefore, we put $I / I_{0}=0.2$ into Eq. (9). The parameter $\beta$ should be close to unity so we put $\beta=0.88$. The onset resistance $R_{0}$ was taken from experiment. The exponent $p$ consists of the temperature dependences of critical field $H_{\mathrm{c}}$ and the coherence length $\xi$, e.g. as follows: $U(T) \propto H_{\mathrm{c}}^{2} \xi \propto g^{*}(T)[1,7]$. That is why an analytical temperature dependence of $g^{*}(t)$ is hardly known so that parameter $p$ shall be variable rather than constant and equal to $1 / 2$. It was impossible to get good fittings with $p=1 / 2$. We carried out the several fitting for different $p$ and we selected $p=1.8$ for $H \| a-b$ plane and $p=2.1$ for $H \| c$ axis. Then we allow two parameters $A^{*}$ and $T_{\mathrm{c}}$ to be varied in order to obtain the best fit. We will interpret our data in terms of Eq. (5) and Eq. (9).

\section{Results and discussion}

These two models mentioned above were used to fit the measurements of the resistance as a function of the temperature for different dc magnetic fields for the $c$-axis oriented, $a-b$ plane aligned $\left(\mathrm{Tl}_{0.6} \mathrm{~Pb}_{0.24} \mathrm{Bi}_{0.16}\right)\left(\mathrm{Ba}_{0.1} \mathrm{Sr}_{0.9}\right)_{2} \mathrm{Ca}_{2} \mathrm{Cu}_{3} \mathrm{O}_{y}$ film on (100) single-crystalline lanthanum aluminate substrate. The film was prepared using screen printing method and the details of preparation and structural analysis are described in the papers $[2,9]$. The X-ray diffraction showed the high purity of the sample and tetragonal structure. The optical micrograph in polarized light displayed that practically all the crystallites are aligned with the $c$-axis perpendicular to the surface of the substrate. The microstructure of the surface was also taken from the scanning electron micrograph. The temperature dependences of resistance $R(T)$ are shown in Figs. 1 and 2. The critical temperature of this superconductor is $T_{\mathrm{c}(R=50 \%)}=111.6 \mathrm{~K}$ in zero applied magnetic field. There is no apparent resistance maximum below $T_{\mathrm{c}}$ due to superconducting weak link because of the very good $a-b$ plane alignment of the crystallites. The critical current densities are: $J_{\mathrm{c} 0}=464 \pm 14 \mathrm{kA} / \mathrm{cm}^{2}$ for $H \| a-b$ and $J_{\mathrm{c} 0}=460 \pm 5 \mathrm{kA} / \mathrm{cm}^{2}$ for $H \| c$ [2].

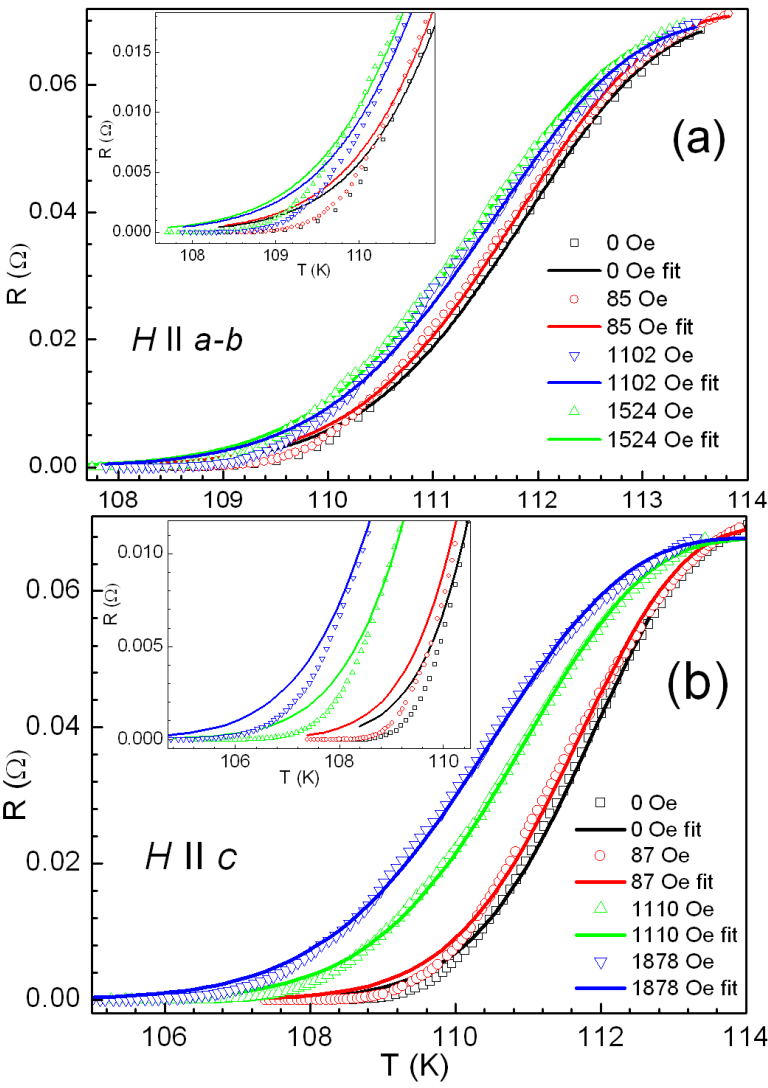

Fig. 1. Resistance versus temperature of a $\left(\mathrm{Tl}_{0.6} \mathrm{~Pb}_{0.24} \mathrm{Bi}_{0.16}\right)\left(\mathrm{Ba}_{0.1} \mathrm{Sr}_{0.9}\right)_{2} \mathrm{Ca}_{2} \mathrm{Cu}_{3} \mathrm{O}_{y}$ film for (a) magnetic fields $H \| a-b$ and (b) magnetic field $H \| c$ [2]. Solid lines are the fitting of Eq. (5). Insets shows $R(T)$ close to $T_{\mathrm{c} 0}$ in enlarged scale.

The fittings using Bessel function (5) are shown in Fig. 1a and b. The fitting parameters for $H \| a-b$ and for $H \| c$ are collected in Tables I and II, respectively.

The fittings using the Anderson and Kim theory represented by Eq. (9) are shown in Fig. 2a and b. Finally, the following parameters in Eq. (9) were fixed: for $H \| a-b$ they are: $I / I_{0}=0.2, p=1.8$ and $\beta=0.88$. For $H \| c$ they are: $I / I_{0}=0.2, p=2.1$ and $\beta=0.88$. In another way it was impossible to get any reasonable fit. The fitting parameters for $H \| a-b$ and for $H \| c$ are collected in Tables III and IV, respectively.

These fittings are quite good except the data close to $T_{\mathrm{c} 0}$. The fits using Eq. (9) describe the measurements of the resistance a bit better than the fits using Eq. (5). The reason is most likely because the Anderson and Kim theory includes the current dependence of activation en- 

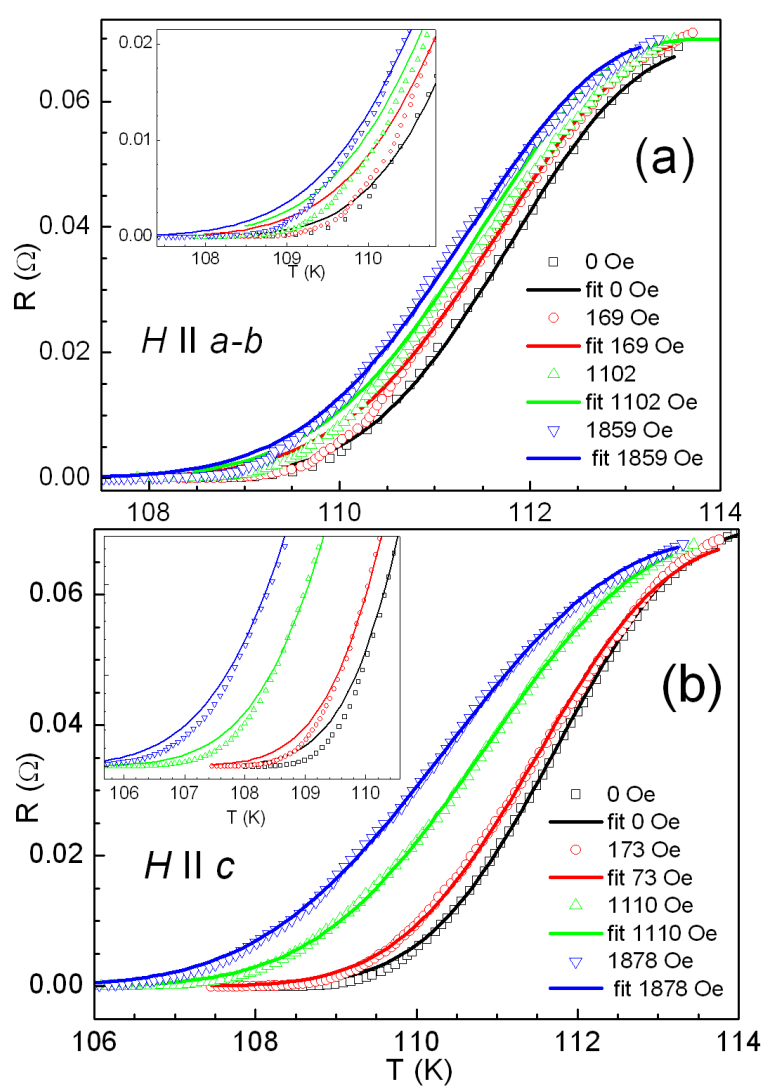

Fig. 2. Resistance versus temperature of a $\left(\mathrm{Tl}_{0.6} \mathrm{~Pb}_{0.24} \mathrm{Bi}_{0.16}\right)\left(\mathrm{Ba}_{0.1} \mathrm{Sr}_{0.9}\right)_{2} \mathrm{Ca}_{2} \mathrm{Cu}_{3} \mathrm{O}_{y}$ film for (a) magnetic fields $H \| a-b$ and (b) magnetic field $H \| c$ [2]. Solid lines are the fitting of Eq. (9). Insets shows $R(T)$ close to $T_{\mathrm{c} 0}$ in enlarged scale.

\section{TABLE I}

Fit parameters $T_{\mathrm{c}}$ and $A$ for the temperature dependence of the resistance according to Eq. (5) for different values of the applied magnetic field for $H \| a-b$.

\begin{tabular}{c|c|c}
\hline \hline$H[\mathrm{Oe}]$ & $T_{\mathrm{c}}[\mathrm{K}]$ & $A[\mathrm{Oe}]$ \\
\hline 0 & $114.4 \pm 0.1$ & $6796 \pm 339$ \\
85 & $114.4 \pm 0.1$ & $7.9 \pm 0.3$ \\
1102 & $114.2 \pm 0.1$ & $0.57 \pm 0.02$ \\
1524 & $114.1 \pm 0.1$ & $0.415 \pm 0.013$
\end{tabular}

TABLE II

As Table I for $H \| c$.

\begin{tabular}{c|c|c}
\hline \hline$H[\mathrm{Oe}]$ & $T_{\mathrm{c}}[\mathrm{K}]$ & $A[\mathrm{Oe}]$ \\
\hline 0 & $114.6 \pm 0.1$ & $6073 \pm 222$ \\
87 & $114.6 \pm 0.1$ & $6.5 \pm 0.3$ \\
1110 & $114.3 \pm 0.1$ & $0.394 \pm 0.009$ \\
1878 & $114.3 \pm 0.1$ & $0.199 \pm 0.005$
\end{tabular}

TABLE III

As Table I for Eq. (9) and $H \|$ $a-b$.

\begin{tabular}{c|c|c}
\hline \hline$H[\mathrm{Oe}]$ & $T_{\mathrm{c}}[\mathrm{K}]$ & $A^{*}[\mathrm{Oe}]$ \\
\hline 0 & $114.2 \pm 0.2$ & $0.735 \pm 0.018$ \\
169 & $114.0 \pm 0.1$ & $346.4 \pm 15.6$ \\
1102 & $114.0 \pm 0.1$ & $1634 \pm 87$ \\
1859 & $113.8 \pm 0.1$ & $2437 \pm 133$
\end{tabular}

TABLE IV

As Table I for Eq. (9) and $H \| c$.

\begin{tabular}{c|c|c}
\hline \hline$H[\mathrm{Oe}]$ & $T_{\mathrm{c}}[\mathrm{K}]$ & $A^{*}[\mathrm{Oe}]$ \\
\hline 0 & $114.9 \pm 0.1$ & $0.976 \pm 0.195$ \\
173 & $114.9 \pm 0.1$ & $604.6 \pm 18.1$ \\
1110 & $114.8 \pm 0.1$ & $1748 \pm 35$ \\
1878 & $114.7 \pm 0.1$ & $2110 \pm 48$
\end{tabular}

ergy. In addition it describes the experimental data for epitaxial and single crystal samples in which the flowing current can be closer to its critical value than in the bulk ones. One can notice that the values of critical temperatures $T_{\mathrm{c}}$ are almost the same and they are very close to the onset temperature $T_{\mathrm{c}}^{\text {onset }}=115.2 \mathrm{~K}$ that is the same for all measured transitions. This temperatures can be stated as a freezing temperature $T_{\mathrm{g}}$ into superconducting vortex-glass phase [4]. This temperatures do not practically depend on the applied magnetic field. The fitting procedures show that the parameters $A$ as well as $A^{*}$ strongly depend on the applied magnetic fields.

In order to get some information about the critical region within the superconducting transition around $T_{\mathrm{c}}$ we calculated the critical exponents of the conductivity according to the papers [10-13]:

$$
\Delta \sigma=K \varepsilon^{-\lambda},
$$

where $\varepsilon=\frac{T-T_{\mathrm{c}}}{T_{\mathrm{c}}}, \lambda$ is the critical exponent, $K$ is a constant, $\Delta \sigma \propto \frac{1}{R}-\frac{1}{R_{0}}$ and $R_{0}$ is the onset resistance where linear dependence starts to develop above $T_{\mathrm{c}}$. To determine the critical exponents we plotted the temperature dependence of the following expression:

$$
-\left[\frac{\mathrm{d}}{\mathrm{d} T} \ln \left(\frac{1}{R}-\frac{1}{R_{0}}\right)\right]^{-1}=\frac{1}{\lambda} \frac{T-T_{\mathrm{c}}}{T_{\mathrm{c}}} .
$$

The critical exponent is a reversal slope of the dependence within a linear region of the temperature. This dependence is shown in Fig. 3.

In HTS, mainly due to the very short coherence length, the short-lived Cooper pairs fluctuate in rather broad temperature region around $T_{\mathrm{c}}$. In contrast in conventional low temperature superconductors there are no fluctuations in accord to the BCS theory. A number of experimental results were analyzed taking into account the fully stochastic Gaussian fluctuations with the exponent $\lambda=2-d / 2$, where $d=1,2$ and 3 is the dimension of the fluctuating system $[12,13]$. Also in our previous pa- 


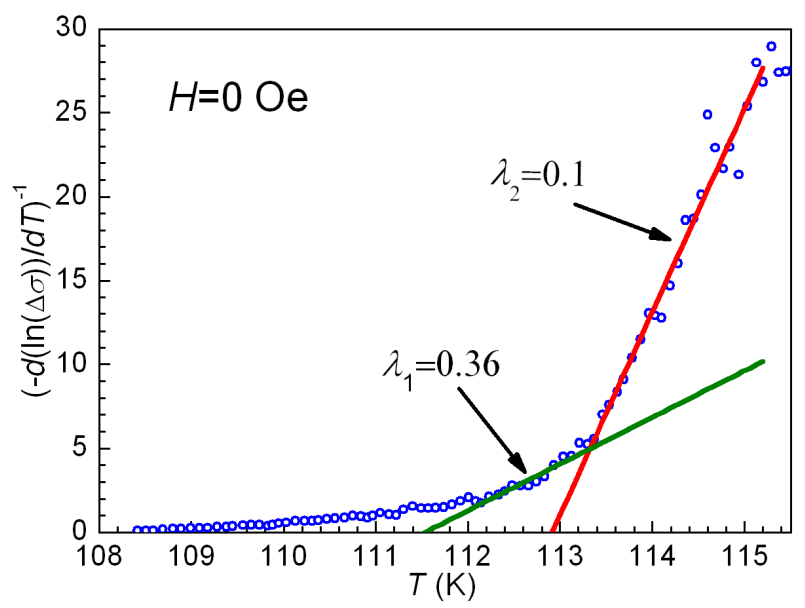

Fig. 3. Logarithmic $T$ derivative of $\Delta \sigma$ as a function of temperature of a $\left(\mathrm{Tl}_{0.6} \mathrm{~Pb}_{0.24} \mathrm{Bi}_{0.16}\right)\left(\mathrm{Ba}_{0.1} \mathrm{Sr}_{0.9}\right)_{2} \mathrm{Ca}_{2} \mathrm{Cu}_{3} \mathrm{O}_{y}$ film at the zero applied magnetic field. The solid lines correspond to fits to Eq. (11). The critical exponents $\lambda_{1}$ and $\lambda_{2}$ are calculated from the fit parameter of Eq. (11).

per [14], on the shape of specific heat anomaly around $T_{\mathrm{c}}$ in $\mathrm{DyBa}_{2} \mathrm{Cu}_{3} \mathrm{O}_{x}$, we found the huge effect of the Gaussian fluctuations within a few Kelvin temperature interval around $T_{\mathrm{c}}$. Since some years also the true critical fluctuations were observed in some HTS very close to $T_{\mathrm{c}}[12,13]$, with the critical exponent $\lambda=\nu(2+z+d+\eta) \approx 1 / 3$ or smaller, where $\nu=2 / 3, z \approx 3 / 2$ and $\eta \approx 0$ [15]. If $\lambda=1 / 3$, then it means that we are dealing with true critical fluctuations which are characteristic for 3-dimensional plane $X Y$ system, called the 3D- $X Y$ model.

In the present paper, in zero applied field, we found $\lambda=0.36 \pm 0.05$ in the closest region above $T_{\mathrm{c}}$ from about $112 \mathrm{~K}$ to $113.5 \mathrm{~K}$ and even much smaller above this region (cf. Fig. 3). Further above this region our phenomenological analysis of the resistance transition according to Eqs. (5) and (9) breaks down. Thus, the analysis of the superconducting thin film is rather confined to the temperature interval where the true critical fluctuations over the Gaussian fluctuations play a dominant role. We leave for future examination the exact analysis of our experimental data on the magnetic field dependent fluctuations.

\section{Conclusions}

The resistive transitions to the superconducting state of the $c$-axis oriented, $a-b$ aligned $\left(\mathrm{Tl}_{0.6} \mathrm{~Pb}_{0.24} \mathrm{Bi}_{0.16}\right)\left(\mathrm{Ba}_{0.1} \mathrm{Sr}_{0.9}\right)_{2} \mathrm{Ca}_{2} \mathrm{Cu}_{3} \mathrm{O}_{y}$ film on (100) single-crystalline lanthanum aluminate substrate were fitted using the Ambegaokar and Halperin as well as the Anderson and Kim theories. The fits using Eq. (9) describe the measurements of the resistance a bit better than the fits using Eq. (5). The reason is most likely because the Anderson and Kim theory includes the current dependence of activation energy. The calculated critical temperatures are very close to the onset temperature $T_{\mathrm{c}}^{\mathrm{onset}}=115.2 \mathrm{~K}$ and can be stated as a freezing temperature $T_{\mathrm{g}}$ into superconducting vortex-glass state. The fitting procedures show that the parameters $A$ as well as $A^{*}$ strongly depend on the applied magnetic fields.

The critical exponent in the closest to $T_{\mathrm{c}}$ temperature interval was determined to be $\lambda=0.36 \pm 0.05$. This means that the analysis of the resistance transition according to Eqs. (5) and (9) of the superconducting thin film is confined to the temperature interval where the true critical fluctuations but not the Gaussian fluctuations play a dominant role according to the 3 -dimensional- $X Y$ plane model of the fluctuations.

\section{Acknowledgments}

This work was supported by the Faculty of Physics and Applied Computer Science, AGH University of Science and Technology, Cracow, Poland. We acknowledge for the sample manufactured in Professor Gritzner's group in the Kepler University in Linz, Austria.

\section{References}

[1] M. Tinkham, Phys. Rev. Lett. 61, 1658 (1988).

[2] W.M. Woch, R. Zalecki, A. Kołodziejczyk, O. Heiml, G. Gritzner, Physica C 434, 17 (2006).

[3] Y. Yeshurun, A.P. Malozemoff, Phys. Rev. Lett. 60, 2202 (1988).

[4] R.H. Koch, V. Foglietti, W.J. Gallagher, G. Koen, A. Gupta, M.P.A. Fisher, Phys. Rev. Lett. 63, 1511 (1989).

[5] V. Ambegaokar, B.I. Halperin, Phys. Rev. Lett. 22, 1364 (1969).

[6] P.W. Anderson, Y.B. Kim, Rev. Mod. Phys. 36, 39 (1964).

[7] Ju-Jin Kim, Ho-kyun Lee, Jinwook Chung, Hyun Joon Shin, $\mathrm{Hu}$ Jong Lee and Ja Kang $\mathrm{Ku}$, Phys. Rev. B 43, 2962 (1991).

[8] E. Zeldow, N.M. Amer, G. Koren, A. Gupta, R.J. Gambino, M.W. McElfresh, Phys. Rev. Lett. 62, 3093 (1989).

[9] O. Heiml, G. Gritzner, Supercond. Sci. Technol. 15, 956 (2002).

[10] L.G. Aslamazov, A.I. Larkin, Soviet Solid State 10 , 875 (1968); Phys. Lett. 26A, 238 (1968).

[11] A.I. Larkin, A.A. Varlamov, in Superconductivity, Eds. K.H. Bennemann, J.B. Ketterson, Vol. 1, Springer-Verlag Berlin, Heidelberg 2008, p. 369.

[12] P. Pureur, R. Menegotto Costa, P. Rodrigues, Jr., J. Schaf, J.V. Kunzler, Phys. Rev. B 47, 11420 (1993).

[13] R. Menegotto Costa, P. Pureur, M. Gusmao, S. Senoussi, K. Behnia, Phys. Rev. B 64, 214513 (2001).

[14] A. Kozłowski, Z. Tarnawski, A. Kołodziejczyk, J. Chmist, T. Ściężor, R. Zalecki, Physica C 184, 113 (1991).

[15] C.J. Lobb, Phys. Rev. B 36, 3930 (1987). 